\title{
Influencia del enfriamiento de la etapa de cocción sobre las propiedades mecánicas del gres porcelánico
}

\section{A. DE NONI JUNIOR ${ }^{(1,2)}$, D. HOTZA ${ }^{(2)}$, V. CANTAVELLA SOLER ${ }^{(3)}$, E. SANCHEZ VILCHES ${ }^{(3)}$}

(1) Instituto Maximiliano Gaidzinski (IMG) - Cocal do Sul - Brasil

(2) Universidade Federal de Santa Catarina (UFSC) - Florianópolis - Brasil

(3) Instituto de Tecnología Cerámica (ITC) - Castellón - España.

\begin{abstract}
El presente estudio ha sido planteado con el objeto de determinar la influencia del enfriamiento de la etapa de cocción sobre el desarrollo de tensiones residuales y las propiedades mecánicas del gres porcelánico. Para ello se prepararon probetas que fueron sometidas a diferentes tipos de enfriamiento. Las tensiones macroscópicas fueron determinadas con el método de relajación de deformaciones por corte incremental. Las tensiones microscópicas sobre las partículas de cuarzo fueron analizadas por DRX. Se observó que durante el enfriamiento rápido el material desarrolla un perfil de tensiones residuales macroscópicas característico de procesos de templado de vidrios, con el consecuente incremento en la resistencia mecánica. La difracción de rayos $\mathrm{X}$ ha puesto de manifiesto que las partículas de cuarzo se encuentran sometidas a tracción, reforzando también el material. Sin embargo, el cuarzo provoca un deterioro microestructural por la generación y crecimiento de grietas. Este último hecho limita el incremento de la resistencia mecánica, lo cual es especialmente notorio para las elevadas velocidades de enfriamiento que tienen lugar después de la transformación alotrópica del cuarzo.
\end{abstract}

Palabras clave: gres porcelánico, enfriamiento, tensión residual, templado, cuarzo.

Influence of post-fire cooling on porcelain tile mechanical properties

This study was undertaken to determine the influence of post-fire cooling on generation of residual stress and mechanical properties in porcelain tile. The test pieces were prepared and subjected to different types of cooling. Macroscopic stresses were determined by the strain relaxation method with incremental cutting. Microscopic stresses on the quartz particles were analysed by X-ray diffraction (XRD). During fast cooling, a macroscopic residual stress profile, characteristic of tempering processes in glasses, was observed to develop with the ensuing increase in mechanical strength. XRD analysis showed that the quartz particles were subjected to tension, additionally strengthening the material. However, the quartz also gave rise to microstructural deterioration owing to crack generation and growth. Such microstructural deterioration limits the increase in mechanical strength, in particular for the high cooling rates used after the allotropic transformation of quartz.

Keywords: porcelain tile, cooling, residual stress, tempering, quartz.

\section{INTRODUCCIÓN}

\subsection{El gres porcelánico}

Las baldosas cerámicas de gres porcelánico, caracterizadas por su elevada cantidad de fase vítrea y baja porosidad, presentan unas excelentes características técnicas y estéticas. Es un producto formulado básicamente a partir de arcillas, que aportan plasticidad, resistencia mecánica en seco y forman mullita y fase vítrea durante la cocción; feldespatos, que son formadores de fase vítrea a baja temperatura; y cuarzo, que ayuda a la estabilidad térmica y dimensional por ser el constituyente de mayor temperatura de fusión $(1,2,3)$. Además, las fases cristalinas remanentes, provenientes de estos materiales, o cristalizadas durante la cocción, desarrollan un importante papel de refuerzo de la matriz vítrea (2).

Las composiciones de gres porcelánico se formulan y procesan para poder realizar la cocción a una temperatura entre
1180 y $1220^{\circ} \mathrm{C}$ con ciclos de 40 a 60 minutos. La temperatura máxima de cocción viene determinada normalmente por la obtención de la máxima densificación. En este punto la absorción de agua es inferior al $0,5 \%$ y la cantidad de porosidad cerrada es baja. El enfriamiento se hace lo más rápidamente posible, respetando únicamente la zona de transformación alotrópica del cuarzo $\left(573^{\circ} \mathrm{C}\right)$, donde se reduce la velocidad de enfriamiento para evitar la rotura del producto, volviendo en seguida a aumentarla hasta la salida del horno (1). Dada la velocidad de la cocción, las reacciones de fusión, cristalización y disolución no llegan a estados de equilibrio termodinámico. 


\subsection{Desarrollo de tensiones residuales en el gres porcelánico durante la etapa de enfriamiento del ciclo de cocción}

El estado de tensiones que existe en el interior de un material, sin aplicación de cargas externas u otras fuentes de tensión, como gradientes térmicos, es debido a la existencia de tensiones residuales. Las tensiones se clasifican de acuerdo con su rango de actuación: macroscópicas, cuando se manifiestan a lo largo de varios granos del material o por todo su espesor; microscópicas, cuando se manifiestan a través de un grano, o parte de él, entre fases o en granos dispersados en una matriz. Finalmente, existen tensiones que se manifiestan a escala interatómica (4).

\subsubsection{Tensiones macroscópicas}

Las tensiones macroscópicas en el gres porcelánico pueden producirse como consecuencia de que el tramo entre la temperatura máxima de cocción $\left(\mathrm{T}_{\mathrm{c}}\right)$ y la temperatura de transformación del cuarzo coincide, aproximadamente, con la región entre la temperatura de fusión de los feldespatos y la temperatura de transformación de la fase vítrea $\left(\mathrm{T}_{\mathrm{g}}\right)$. En la Figura 1 se detalla este intervalo insertado en una curva típica de cocción del gres porcelánico. Se trataría por lo tanto de un proceso que se asemejaría al de templado del vidrio. Debido a las elevadas velocidades de enfriamiento empleadas y la baja conductividad térmica del material, se establece un gradiente térmico entre la superficie y el interior $(5,6)$. Los materiales sometidos a estas condiciones suelen desarrollar un perfil de tensiones parabólico, siendo la ec. 1 una estimación de la tensión de compresión máxima que puede originarse en la superficie (6):

$$
\sigma_{s}=\frac{-2 \cdot \alpha \cdot E \cdot \Delta T}{3 \cdot(1-v)}
$$

Siendo: $\sigma_{s}$, la tensión residual en la superficie; $\alpha$, el coeficiente de dilatación térmica lineal; E, módulo de elasticidad; $v$, el coeficiente de Poisson; $\Delta T$, la máxima diferencia de temperatura entre el interior y la superficie cuando la capa interior se encuentra en estado rígido.

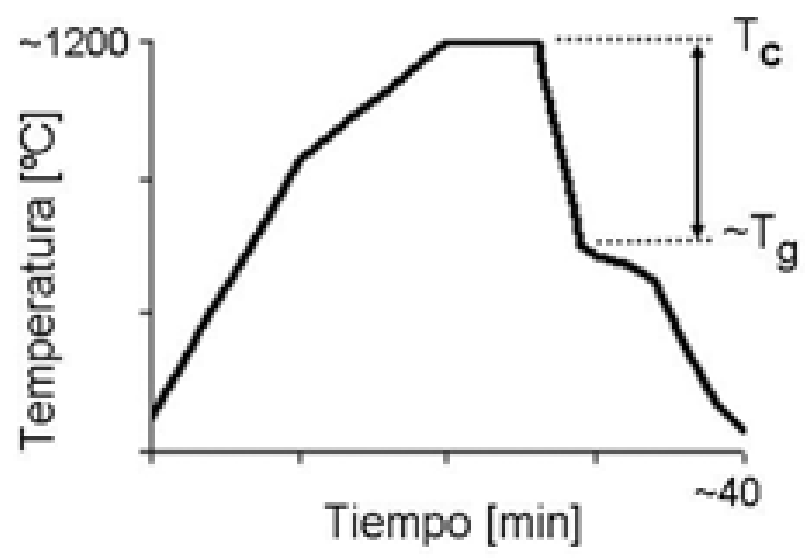

Fig. 1- Indicación del intervalo en el que se podría desarrollar un proceso de templado en un ciclo de cocción industrial de gres porcelánico.
Un producto que desarrolle tensión residual de compresión en una capa de la superficie puede ver incrementada su resistencia mecánica a la flexión de acuerdo con la ec.2 $(5,7)$ :

$$
\sigma_{t a}=\sigma_{o}-\sigma_{s}
$$

Siendo: $\sigma_{t a}=$ resistencia mecánica del producto con tensión residual macroscópica; $\sigma_{O}=$ resistencia mecánica del producto sin tensión residual.

Esta ecuación es válida siempre y cuando se cumplan las siguientes hipótesis: A) el tamaño del defecto natural del producto tensionado sea igual al del producto sin tensiones; B) la tensión de compresión en la superficie sea constante al menos hasta una profundidad equivalente al tamaño del defecto. No obstante, pueden existir piezas tensionadas con un tamaño del defecto más grande, en las que se observe este incremento de resistencia, siempre y cuando la magnitud de las tensiones residuales sea suficientemente elevada.

\subsubsection{Tensiones microscópicas}

En lo que se refiere a las tensiones existentes a escala microscópica, éstas son debidas a la diferencia entre los coeficientes de expansión térmica de las fases cristalinas (c) y de la matriz vítrea (m). Las tensiones que una partícula esférica de un material isótropo ejerce sobre una matriz infinita son descritas de acuerdo con las ecuaciones 3 y $4(8,9)$ :

$$
\sigma_{r r}=\frac{\Delta \alpha \cdot \Delta T^{\prime}}{\frac{1+v_{m}}{2 \cdot E_{m}}+\frac{1-2 v_{c}}{E_{C}}} \cdot\left(\frac{R}{x}\right)^{3}
$$

$$
\sigma_{\theta \theta}=\frac{-\sigma_{r}}{2}
$$

Siendo: $\sigma_{r r}$, la tensión radial; $\Delta \alpha$ diferencia entre los coeficientes de expansión térmica lineal de la partícula y la matriz; $\Delta T$, el intervalo de temperatura del enfriamiento, en el caso de una matriz vítrea, se considera desde la temperatura de transformación vítrea hasta la temperatura ambiente; E, el módulo de elasticidad de la partícula (c) y de la matriz (m); $\mathrm{R}$, radio de la partícula; $x$, la distancia desde el centro de la partícula hasta un punto de la misma; $\sigma_{\theta \theta}$ la tensión tangencial.

Como muestra la ec.3, la tensión radial asume valor máximo en la interfase partícula-matriz. Para el caso del gres porcelánico, las mayores tensiones son aquéllas desarrolladas entre la matriz vítrea y las partículas de cuarzo, ya que el resto de componentes presentan coeficientes de expansión térmica más próximos a la matriz. El cuarzo- $\alpha$, al desarrollar una dilatación más grande que la matriz, da lugar a una tensión radial de tracción en la interfase partícula-matriz y compresión tangencial en la matriz (9). Dependiendo de la magnitud de estas tensiones la partícula puede despegarse de la matriz y generar microgrietas, en este caso las tensiones residuales son aliviadas. La Figura 2 muestra un esquema de los posibles estados de tensiones macroscópicas y microscópicas en la superficie del gres porcelánico. 


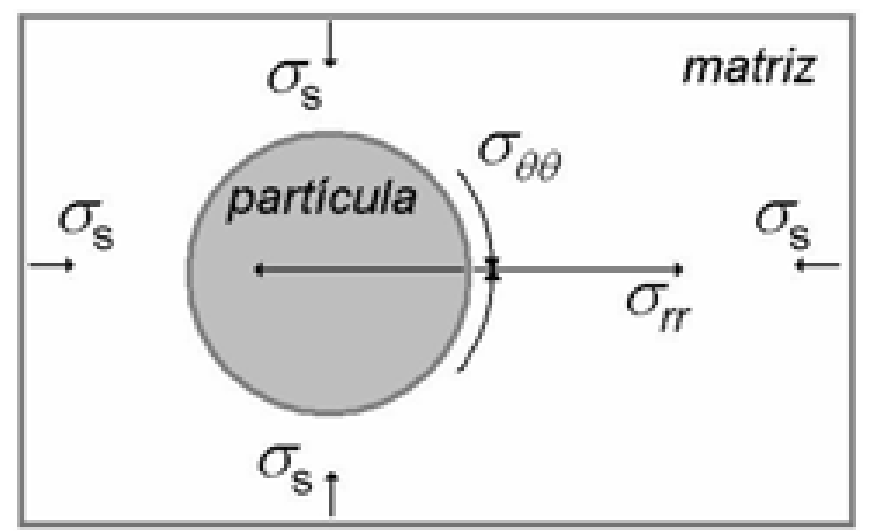

Fig. 2- Posibles estados de tensiones macroscópicas $\left(\sigma_{s}\right)$ y microscópicas $\left(\sigma_{r r}, \sigma_{\theta \theta}\right)$, en la superficie del gres porcelánico.

Numerosos trabajos de investigación con porcelanas triaxiales (9-15), indican que partículas de cuarzo de tamaño comprendido entre $20-30 \mu \mathrm{m}$ son las que producen los máximos valores de resistencia mecánica, por estar aún conectadas con la matriz y seguir sin disolverse tras la cocción. Este intervalo de tamaños de partícula es muy común en las composiciones industriales de gres porcelánico.

Por otro lado, el desarrollo de tensiones residuales también es una importante característica en cerámicas multicapa que contribuye a mejorar las propiedades mecánicas de estos materiales (16). Para la medida de las tensiones residuales en piezas cerámicas existen básicamente dos métodos. El primero está basado en la destrucción del estado de equilibrio interno, de este modo la tensión puede evaluarse a partir de la consecuente relajación. El segundo método, no destructivo, está basado en la relación existente entre las tensiones y los parámetros físicos o cristalográficos de los cristales del material (4).

\subsection{Objeto del estudio}

El presente estudio tiene por objeto evaluar las tensiones residuales, tanto macroscópicas como microscópicas, que se desarrollan sobre piezas de gres porcelánico, asociadas a la etapa de enfriamiento del ciclo de cocción. Así mismo, se ha analizado la influencia que estas tensiones ejercen sobre la resistencia mecánica del producto, para lo cual ha sido necesario considerar el papel que ejerce la microestructura final de la pieza cocida y su dependencia con la velocidad de enfriamiento. Se utilizaron una técnica destructiva y una no destructiva para analizar los distintos estados de tensiones desarrollados en el gres porcelánico.

\section{PROCEDIMIENTO EXPERIMENTAL}

\subsection{Obtención de las probetas a distintas velocidades de enfriamiento}

Se prepararon probetas de $80 \times 20 \times 7 \mathrm{~mm}$ por prensado a partir de un polvo atomizado industrial. El polvo contenía un 5,5\% de humedad (base seca) y se empleó una presión de prensado de $45 \mathrm{MPa}$. Después del secado en estufa a $110{ }^{\circ} \mathrm{C}$, las probetas fueron cocidas en horno eléctrico. Para todas las cocciones la velocidad de calentamiento fue de $70{ }^{\circ} \mathrm{C} / \mathrm{min}$, entre $25-500{ }^{\circ} \mathrm{C}$, y $25{ }^{\circ} \mathrm{C} / \mathrm{min}$, entre $500-1190{ }^{\circ} \mathrm{C}$. El tiempo de permanencia a la temperatura máxima de cocción fue de 6 min. Se escogió $1190^{\circ} \mathrm{C}$ como temperatura máxima, ya que se corresponde con la temperatura de máxima densificación del producto para el ciclo de cocción ensayado.

Las probetas fueran sometidas a siete condiciones de enfriamiento distintos: combinando enfriamientos dentro o fuera del horno (DH o FH respectivamente) y con empleo de convección natural o forzada ( $\mathrm{CN}$ o $\mathrm{CF}$ respectivamente). El objeto de esta serie de experimentos fue modificar principalmente la velocidad de enfriamiento en la zona donde predomina el comportamiento viscoelástico del vidrio (es decir, desde la máxima temperatura de cocción, $1190^{\circ} \mathrm{C}$, hasta $650 \pm 20^{\circ} \mathrm{C}$ ), y en la zona en torno a la transformación alotrópica del cuarzo $\left(<650^{\circ} \mathrm{C}\right)$. La tabla I relaciona los códigos con las características de los tipos de enfriamiento empleados.

TABLA I. CÓDIGOS Y CARACTERÍSTICAS DE LOS TIPOS DE ENFRIAMIENTO EMPLEADOS.

\begin{tabular}{|c|c|c|c|}
\hline Códigos & Posición & $\begin{array}{c}\text { Enfriamiento } \\
1190-680^{\circ} \mathrm{C}\end{array}$ & $\begin{array}{c}\text { Enfriamiento } \\
<650^{\circ} \mathrm{C}\end{array}$ \\
\hline E1 & $\mathrm{DH}$ & $\mathrm{CN}-\mathrm{P}^{1}$ & $\mathrm{CN}$ \\
\hline E2 & $\mathrm{DH}$ & $\mathrm{CF}-\mathrm{H}^{2}$ & $\mathrm{CF}$ \\
\hline E3 & $\mathrm{FH}$ & $\mathrm{CN}-8$ & $\mathrm{CN}$ \\
\hline E4 & $\mathrm{FH}$ & $\mathrm{CN}-1$ & $\mathrm{CN}$ \\
\hline E5 & $\mathrm{FH}$ & $\mathrm{CF}-\mathrm{V}$ & $\mathrm{CN}$ \\
\hline $\mathrm{E} 6$ & $\mathrm{FH}$ & $\mathrm{CF}-\mathrm{VA}$ & $\mathrm{CN}$ \\
\hline E7 & $\mathrm{FH}$ & \multicolumn{2}{|c|}{ CF-VA } \\
\hline
\end{tabular}

1 Sistema de ventilación del horno apagado; 2 Sistema de ventilación del horno encendido

Los enfriamientos fuera del horno (FH) se llevaron a cabo utilizando un montaje que se detalla en la Figura 3. La placa refractaria con 8 probetas (P1) era sacada del horno a la temperatura de cocción y puesta en un soporte refractario (P2) equipado con dos ventiladores (P3) y una distribución de aire comprimido (P4). El enfriamiento E3 se hizo simplemente por convección natural ( $\mathrm{CN}-8$, con 8 probetas); para el E5 se accionaron únicamente los ventiladores (CF-V) durante el primer tramo del enfriamiento; E6 y E7 se llevaron a cabo con los ventiladores y con el aire comprimido (CF-VA), aunque con el E7 no se interrumpió la convección forzada a lo largo de todo el enfriamiento. El enfriamiento E4 se realizó fuera del horno pero sacando una probeta cada vez para enfriar por convección natural $(\mathrm{CN}-1)$, siendo por lo tanto más rápido que el E3 (CN-8), ya que no se encuentra sujeto a la inercia térmica de la placa refractaria que contenía las 8 probetas. En resumen, podemos hablar de dos grupos distintos de enfriamiento; el grupo 1:E1, E2, E3, E4; y el grupo 2: E5, E6, E7. La diferencia es que en el primer grupo las perdidas de calor son menores por no haber convección forzada. La siguiente secuencia relaciona, en orden creciente, los tipos de enfriamiento ensayados según el nivel de severidad, tanto para la generación de tensiones macroscópicas, como para el deterioro microestructural asociado a la transformación del cuarzo:

$\mathrm{E} 1<\mathrm{E} 2<\mathrm{E} 3<\mathrm{E} 4<\mathrm{E} 5<\mathrm{E} 6<\mathrm{E} 7$

El enfriamiento industrial sería aproximadamente comparable al enfriamiento E5. 


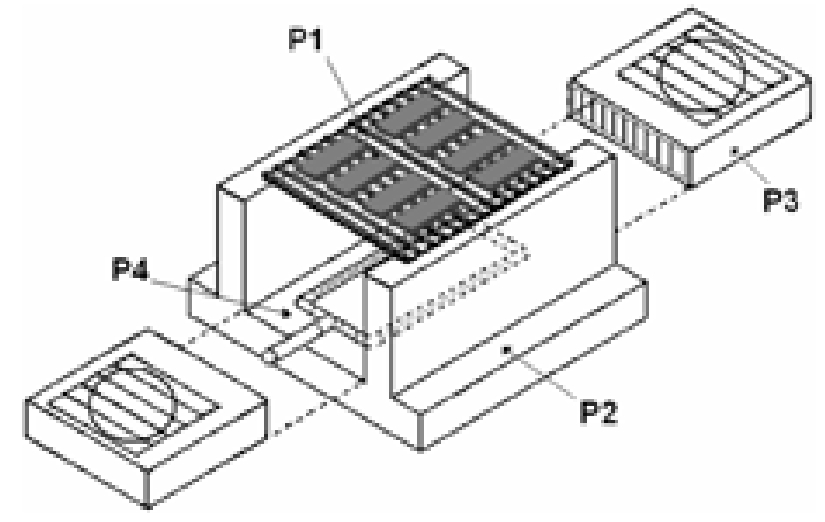

Fig. 3- Montaje diseñado para realizar los enfriamientos rápidos fuera del horno, compuesto por una placa refractaria y probetas (P1), un suporte refractario (P2), dos ventiladores laterales (P3) y un distribuidor de aire comprimido (P4).

\subsection{Caracterización de las probetas}

Se realizaron ensayos de resistencia mecánica por flexión en tres puntos de apoyo con doce probetas para cada tipo de enfriamiento, preparadas tal como lo descrito en el apartado 2.1. Para ello se ha utilizando una máquina universal de ensayos mecánicos (Instron 6027) con célula de carga de 1 $\mathrm{kN}$ y velocidad de aplicación de $1 \mathrm{~mm} / \mathrm{min}$. Algunas de las probetas fueron examinadas por Microscopia Eletrónica de Barrido (MEB) con el objeto de inspeccionar la microestructura del material en zonas transversales, próximas a la superficie.

\subsection{Medida de las tensiones residuales}

\subsubsection{Método de relajación de deformaciones por corte incremental}

La técnica, empleada también para vidrios metálicos (17), consiste en adherir una galga extensiométrica a la probeta que se quiere analizar, realizar un entalla de una determinada profundidad $\left(a_{i}\right)$ y medir la deformación que sufre la zona de la galga tras el corte $\left(\varepsilon_{g i}\right)$. La Figura 4 muestra el esquema experimental del método. La deformación libre $\left(\varepsilon_{f}\right)$ dentro de la probeta se puede descomponer como una combinación lineal de polinomios de Legendre $\left(P_{k}\right)$ :

$$
\varepsilon_{f}=\sum_{k} \lambda_{k} \cdot P_{k}(\zeta)
$$

Siendo: $\zeta$, la coordenada en la dirección del espesor; $(\zeta=-1)$ para la superficie inferior y $(\zeta=+1)$ para la superficie superior y $\lambda_{k}$ una constante.

Las constantes $\lambda^{k}$ se calculan a partir de los valores $\left(a_{i}, \varepsilon_{g i}\right)$ con ayuda ${ }^{\prime}$ de unas "funciones de calibrado" determinadas teóricamente mediante un cálculo previo por elementos finitos, y que son función de la geometría de las probetas y posición relativa entalla / galga extensiométrica. A partir de $\lambda_{k}$ se puede calcular directamente el perfil de tensiones macroscópicas dentro de una pieza, $\sigma(\zeta)$

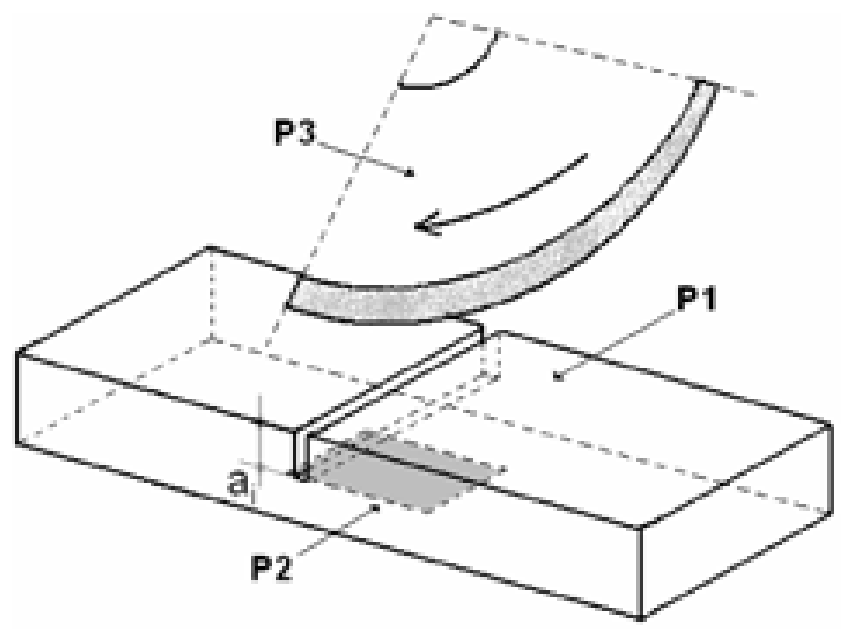

Fig. 4- Esquema experimental del método de relajación de deformaciones por corte incremental: probeta (P1), galga (P2), disco de corte (P3)

Con una probeta de cada tipo de enfriamiento se midieron las tensiones residuales de la superficie mediante este método. Para ello se empleó un disco de corte de cobre diamantado de espesor $0,4 \mathrm{~mm}$ y galgas extensiométricas rectangulares (HBM de $3 \mathrm{~mm})$. Las diferencias de temperatura entre el interior y la superficie de las probetas fueran calculadas a partir de la ecuación 1 (considerando: $\alpha=50 \times 10^{-7{ }^{\circ}} \mathrm{C}^{-1}, \mathrm{E}=70 \mathrm{GPa} v=0,2$ ). Las velocidades de enfriamiento entre $650-500^{\circ} \mathrm{C}$ fueran medidas con una termocámara (Flir E25), con lentes especiales para temperaturas hasta $900^{\circ} \mathrm{C}$.

\subsubsection{Método de difracción de rayos $X$.}

$\mathrm{Su}$ principio está basado en la determinación del desplazamiento de los picos de difracción como consecuencia de la deformación de la red cristalina. Aplicando la ley de Bragg se puede determinar la deformación relativa de un plano (hkl), $\varepsilon_{\mathrm{hkl}^{\prime}}$ de acuerdo con la ec. 6 (4):

$$
\varepsilon_{h k l}=-0,5 \cdot \Delta(2 \theta) \cdot \cot (\theta)=\frac{d_{h k l}^{t i}-d_{h k l}^{o}}{d_{h k l}^{o}}
$$

Siendo: $\Delta(2 \theta)$, el desplazamiento del pico de difracción; $\theta$; el ángulo de difracción del material sin tensiones; $d_{h k l^{\prime}}^{t i}$ la distancia interplanar del material tensionado; $d_{h k l}^{o}$, la distancia interplanar del material sin tensiones. De este modo la estructura cristalina del material es utilizada como una galga extensiométrica absoluta.

De acuerdo con la geometría de la estructura cristalina (18, 19) cabe esperar un aumento de la distancia interplanar para las cuatro familias de planos del cuarzo responsables de los tres picos de difracción característicos de este componente. Las difracciones de rayos $X$ se llevaron a cabo con un difractómetro (Broker AXS, D8 advance) empleando la radiación $\mathrm{K} \alpha 1 \mathrm{Cu}$, a un tiempo de lectura de 1 s para cada $0,01^{\circ}$ entre $10^{\circ}$ y $70^{\circ}(2 \theta)$, y patrón interno de corindón 1976-NIST. El desplazamiento del pico del cuarzo se determinó para el plano [112]. 


\section{RESULTADOS Y DISCUSIÓN}

3.1 Análisis de la tensión residual macroscópica en superficie y su influencia sobre la resistencia mecánica del gres porcelánico.

La Tabla II muestra los resultados obtenidos para la resistencia mecánica a la flexión $\left(\sigma_{t a}\right)$, tensión residual en la superficie $(\sigma)$, diferencia de temperatura entre el interior y la superficie $(\Delta T$, ec.1), velocidad de enfriamiento entre 650$500^{\circ} \mathrm{C}\left(\mathrm{v}_{\mathrm{q}}\right)$ y el desplazamiento de la distancia interplanar del cuarzo $\left(\varepsilon_{112}\right)$.

TABLA II. RESULTADOS OBTENIDOS EN LOS ENSAYOS REALIZADOS CON DIFERENTES VELOCIDADES DE ENFRIAMIENTO

\begin{tabular}{|c|c|c|c|c|c|}
\hline Enfri. & $\begin{array}{c}\sigma_{t a} \\
{[\mathrm{MPa}]}\end{array}$ & $\begin{array}{c}\sigma_{s} \\
{[\mathrm{MPa}]}\end{array}$ & $\begin{array}{c}\Delta T \\
{\left[{ }^{\circ} \mathrm{C}\right]}\end{array}$ & $\begin{array}{c}\mathrm{V}_{\mathrm{q}}^{*} \\
{\left[{ }^{\circ} \mathrm{C} / \mathrm{min}\right]}\end{array}$ & $\begin{array}{c}\varepsilon_{112} \\
{[\%]}\end{array}$ \\
\hline E1 & $68,2 \pm 2.5$ & 0 & $\sim 0$ & 5,5 & 0,22 \\
\hline E2 & $69,9 \pm 1.8$ & 1,7 & 7 & 35 & 0,22 \\
\hline E3 & $73,6 \pm 2.4$ & 7,2 & 58 & 240 & 0,21 \\
\hline E4 & $80,3 \pm 1.8$ & 14,3 & 31 & 220 & 0,18 \\
\hline E5 & $75,0 \pm 1.5$ & 11,2 & 48 & 270 & 0,21 \\
\hline E6 & $82,2 \pm 1.0$ & 22,8 & 98 & 270 & 0,23 \\
\hline E7 & $76,3 \pm 3.0$ & 17,0 & 73 & 500 & 0,20 \\
\hline
\end{tabular}

${ }^{*}$ Error 10\%

En primer lugar puede observarse que, a excepción del enfriamiento menos enérgico (E1), en el resto de los casos las probetas presentan tensiones residuales macroscópicas de compresión en su superficie. En efecto, las elevadas velocidades de enfriamiento, junto con la baja conductividad térmica del gres porcelánico dan lugar a que las capas exteriores se enfríen más deprisa, alcancen su rigidez y contraigan antes que las interiores, todavía calientes y por tanto más deformables. Cuando las capas interiores se hacen rígidas ya no pueden contraer libremente debido a la resistencia de las capas exteriores, lo que da lugar a gradientes de tensiones. Mientras se mantiene el gradiente térmico, las capas interiores se encuentran sometidas a compresión y las exteriores a tracción. Cuando el gradiente térmico desaparece, la mayor contracción de la parte central provoca una tensión de tracción en esta zona, y de compresión en la superficie (6).

Como se puede observar en la Tabla II, la máxima tensión residual $(22,8 \mathrm{MPa})$ se ha obtenido con uno de los enfriamientos más rápidos (E6). En estas mismas condiciones, un vidrio flotado desarrollaría una tensión residual de aproximadamente 50-60 $\mathrm{MPa}$, lo que pone de manifiesto la importancia del valor de la tensión residual obtenido para el gres porcelánico.

En la Figura 5 se representa el incremento de la resistencia mecánica $\left(\sigma_{t a}-\sigma_{o}\right)$ en función de la tensión residual en la superficie $\left(\sigma_{s}\right)$. La línea corresponde al valor teórico que cabría esperar a partir de la ec. 2. Dado que la tensión residual en la superficie es de compresión, se observa un incremento de la resistencia mecánica en todos los casos. No obstante, el comportamiento entre los dos grupos de enfriamientos (1 y 2 según apartado 2.1) es bien distinto, tal como se refleja en los bloques de puntos destacados en el gráfico. Para los enfriamientos del grupo 1 (E1 a E4), en los que no hay convección forzada, los valores se acercan más a los teóricos que en los casos del grupo 2 (E5 a E7), en los que la contribución del mecanismo de convección es mucho más importante. Atendiendo a los valores de $\mathrm{v}_{\mathrm{q}}$ se debe tener en cuenta que el segundo grupo presenta mayor velocidad de enfriamiento en el tramo de la transformación alotrópica del cuarzo que el primero. Como consecuencia de ello, la diferencia de temperatura originada en la primera parte del enfriamiento, $\Delta T$, se mantiene en cierta magnitud, dando lugar a mayores gradientes térmicos en el momento de la transformación del cuarzo, sobre todo en la superficie; y presumiblemente, tal como se describe ampliamente en la bibliografía (9-15), un mayor deterioro microestructural. Estos resultados parecen indicar que conforme más grande es la tracción en la superficie en el intervalo de temperatura donde tiene lugar la transformación alotrópica del cuarzo, más fácilmente se generan defectos microestructurales asociados a dicha transformación. Este deterioro microestructural, analizado más adelante, es la causa de las desviaciones observadas respecto de los valores teóricos.

Finalmente, puede observarse que el punto correspondiente al enfriamiento más enérgico, E7, se encuentra por debajo del E6. Esta anomalía viene motivada por el hecho de que este enfriamiento es el único en el que se ha mantenido la convención forzada durante el tramo de transformación alotrópica del cuarzo $\left(\mathrm{v}_{\mathrm{q}}=500{ }^{\circ} \mathrm{C} / \mathrm{min}\right)$, lo que afectó severamente a la microestructura de las piezas cocidas. De hecho, durante la medida de la tensión residual macroscópica, las probetas rompían con facilidad.

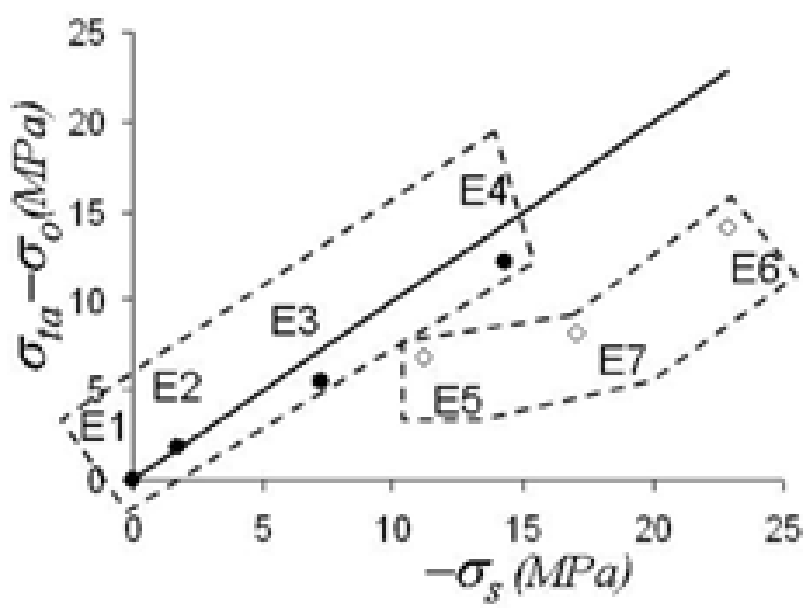

Fig. 5- Incremento de la resistencia mecánica $\left(\sigma_{t a}-\sigma_{o}\right)$ en función de la tensión residual de superficie $\left(\sigma_{s}\right)$; probetas pertenecientes al grupo $1(\bullet)$ y al grupo $2(\diamond)$ de los enfriamientos. La línea indica los valores teóricos según la ecuación 2 .

\subsection{Análisis de las tensiones microscópicas en las partículas de cuarzo}

Los difractogramas obtenidos mostraron en todos los casos las fases cristalinas características del gres porcelánico: cuarzo, mullita y albita no fundida $(1,2)$. Particularmente, para el caso del cuarzo, los picos del plano [112] se encuentran desplazado entre $0,18 \%$ y $0,22 \%$. Como se indicó en el apartado 1.2.2, las partículas de cuarzo, que se encuentran sometidas a tensión de tracción, producen una compresión tangencial sobre la matriz, lo que genera efecto de refuerzo. A partir de estos 
valores de deformación, el cálculo de la tensión sobre este plano, aplicando la ley de Hooke, nos conduce a valores comprendidos entre 140-170 MPa. Sin embargo, utilizando la ec.3, se obtienen valores casi cuatro veces superiores (520-620 MPa). Existen básicamente dos motivos para estas discrepancias:

(A) La ec. 3 no considera que haya influencia de partículas próximas (asume que la matriz es infinita), lo que puede ser válido para fracciones volumétricas de cristales hasta un 15\%. Sin embargo, para el gres porcelánico, la fracción volumétrica, sumando todas las fases cristalinas, normalmente esta comprendida entre $30-45 \%$, de las cuales $15-25 \%$ son de partículas de cuarzo. No obstante, se estima que este factor no influye más allá del 5\%.

(B) La gran diferencia entre los coeficientes de expansión térmica del cuarzo con respecto a la matriz, que es probablemente la principal causa. Esta diferencia de expansión térmica genera un importante deterioro microestructural en forma de grietas alrededor de las partículas de cuarzo durante el enfriamiento, tal como se indica en la bibliografía (9-15), dando lugar a una relajación parcial de la estructura cristalina. Este deterioro microestructural, a diferencia de lo indicado en el apartado anterior, es independiente de la velocidad de enfriamiento, tal como refleja la escasa relación existente entre el desplazamiento planar del cuarzo y la tensión residual macroscópica (Tabla II).

En la Figura 6 se propone un modelo explicativo del deterioro microestructural. La diferencia de expansión térmica entre el cuarzo y la matriz vítrea genera, independientemente de la velocidad de enfriamiento, grietas periféricas alrededor de las partículas de cuarzo. Al aumentar la velocidad de enfriamiento, se acentúa el desarrollo de un perfil de tensiones térmicas que asume valores máximos en la superficie, dando lugar a las condiciones adecuadas para que estas grietas crezcan. En casos extremos, y para tamaños de partícula de cuarzo grandes, se podría llegar a un desprendimiento de las partículas de la matriz. En estos últimos casos, se produciría una relajación de las tensiones microscópicas, hecho no observado en la presente investigación.
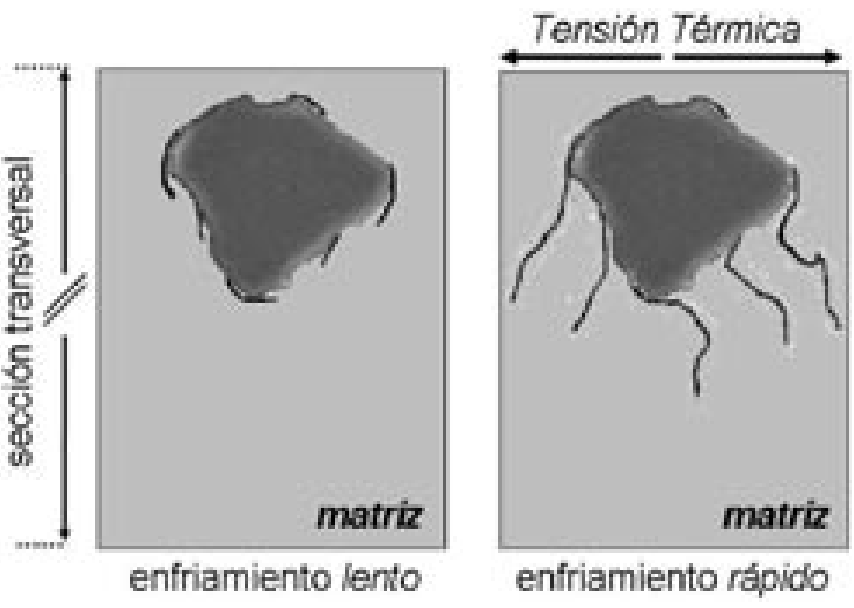

Fig. 6- Modelo explicativo del deterioro microestructural que tiene lugar durante el enfriamiento del gres porcelánico.

\subsection{Análisis microestructural}

Las dos micrografías presentadas en la Figura 7 son representativas de los enfriamientos E1 y E6. Las microestructuras observadas son típicas del gres porcelánico (1), o sea, matriz vítrea, partículas cristalinas embebidas en la matriz vítrea, poros y algunos desconchados y desprendimientos de partícula provenientes del proceso de pulido para la preparación de las probetas. En ambos casos se observa que gran parte de las partículas de cuarzo se encuentran agrietadas. Este fenómeno puede ser debido en parte también al método de preparación de las probetas, tal como recogen algunos investigadores (10). Sin embargo, tampoco sería descartable que parte de estas grietas tengan su origen en el elevado esfuerzo de tracción al que se encuentran sometidas las partículas de cuarzo y que se puso de manifiesto en el apartado anterior.

Por otro lado se observa, que para partículas de cuarzo de un tamaño superior a aproximadamente $5 \mu \mathrm{m}$, empiezan a generarse grietas periféricas de intensidad creciente a medida que aumenta el tamaño de las partículas. Estas grietas pueden prolongarse en la matriz, tal como se ha postulado en el modelo de la Figura 6. Desgraciadamente, la observación microscópica de este crecimiento de grieta resulta muy compleja.

Con el objeto de solventar esta dificultad y tratar de
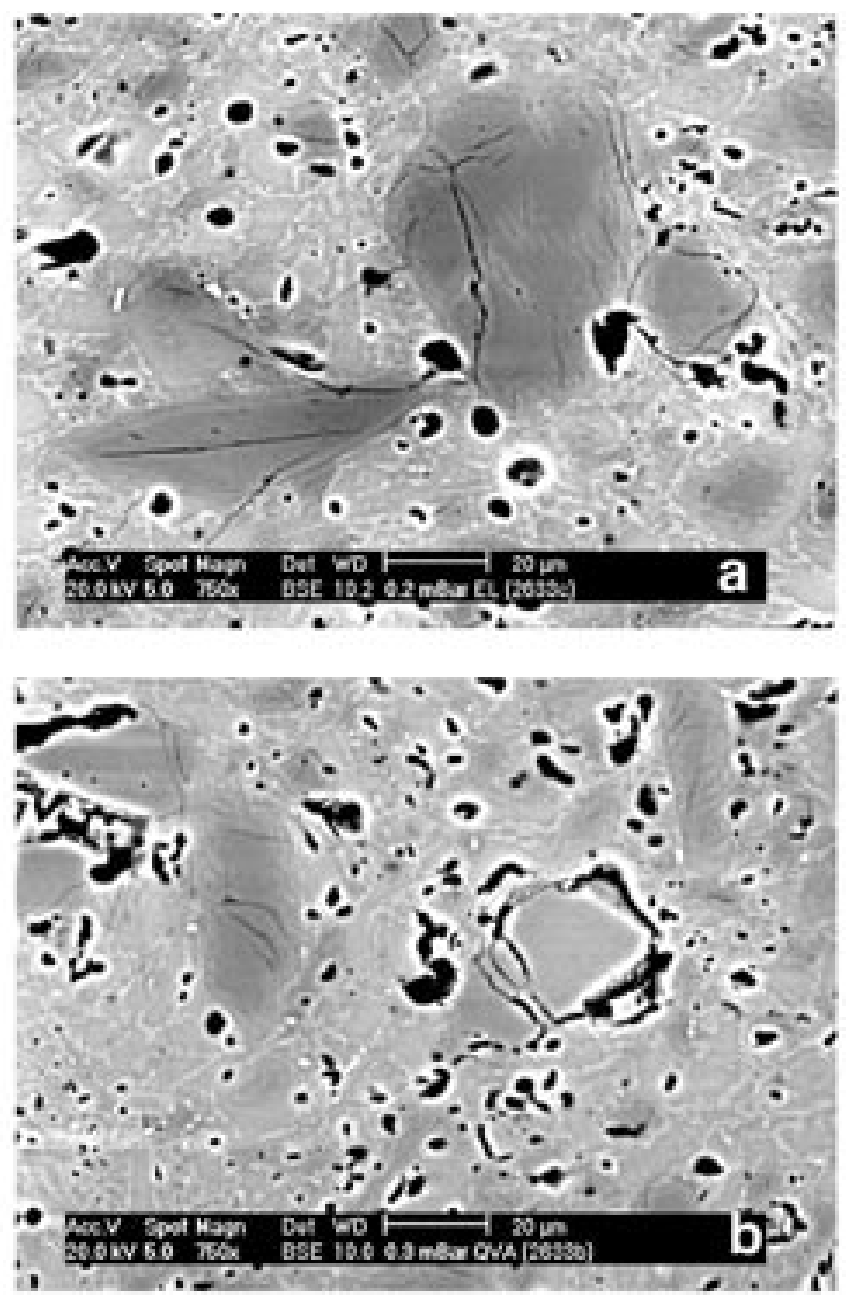

Fig. 7- Micrografías en MEB de probetas correspondientes a enfriamiento lento E1(a) y enfriamiento rápido E6 (b). 
cuantificar el deterioro microestructural asociado a la distinta velocidad de enfriamiento, se realizó un análisis de imágenes en las probetas correspondientes a los dos enfriamientos anteriores (E1 y E6). Para ello se inspeccionó una superficie de $12 \times 10^{4} \mu \mathrm{m}^{2}$ de cada probeta a 300 aumentos, en el microscopio electrónico (Figura 8). Se midió el área ocupada por la porosidad + desconchados $(\mathrm{AP}+\mathrm{AD})$ (regiones más oscuras de las imágenes en la Figura 8) y la distribución de tamaño
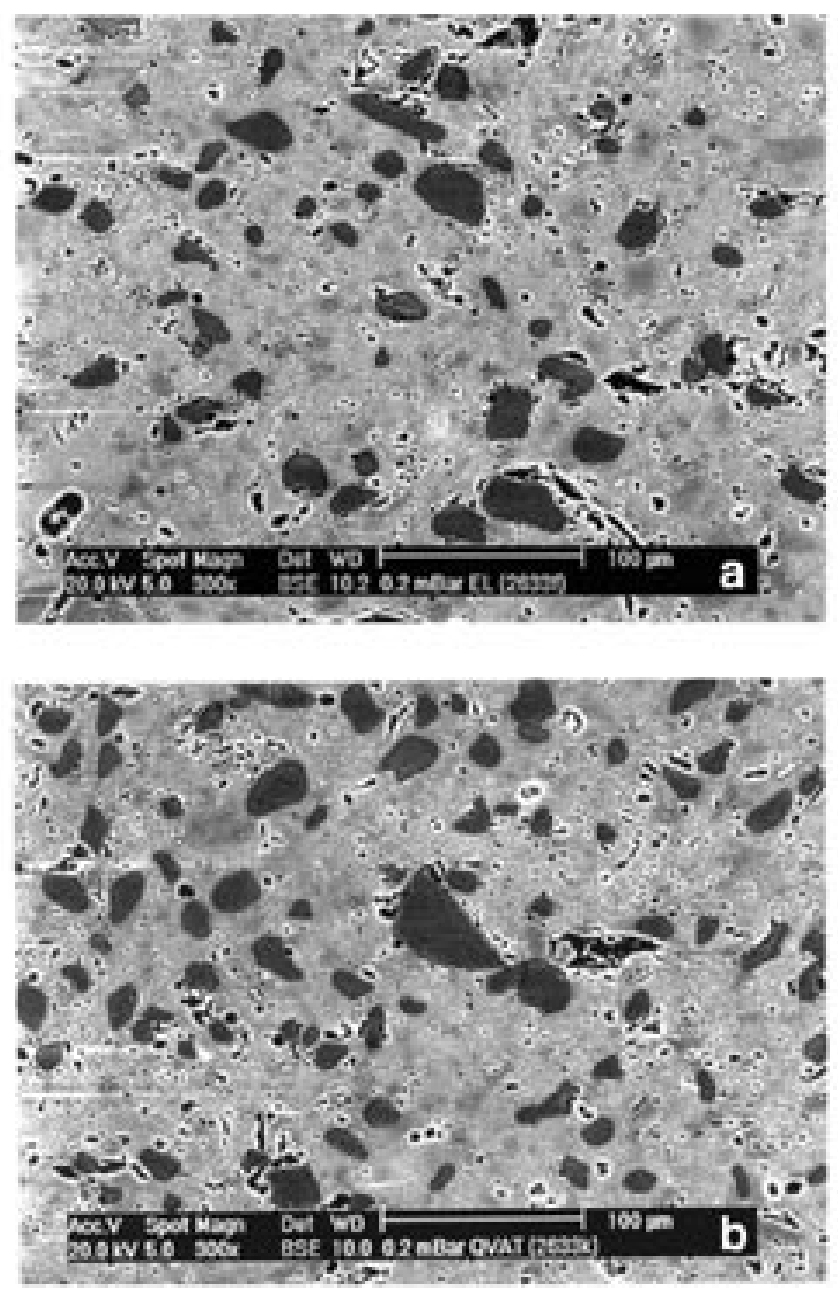

Fig. 8- Regiones cuantificadas por análisis de imágenes de probetas correspondientes a: (a) Enfriamiento lento E1 y (b) enfriamiento rápido E6.

TABLA III. RESULTADO DEL ANÁLISIS DE IMÁGENES REALIZADO CON PROBETAS CORRESPONDIENTES AL ENFRIAMIENTO LENTO (E1) Y ENFRIAMIENTO RÁPIDO (E6)

\begin{tabular}{|c|c|c|}
\hline Propiedad & E1(lento) & E6(rápido) \\
\cline { 1 - 2 } AP+AD $(\%)$ & 6,2 & 6,5 \\
\cline { 1 - 2 } A-CGM $(\%)$ & 8,4 & 15,3 \\
\cline { 1 - 2 } DTP & \multicolumn{2}{|c|}{ Frecuencia acumulativa porcentual } \\
\cline { 1 - 2 } Diámetro $(\mu \mathrm{m})$ & 0 & 0 \\
\hline 5 & 20 & 62 \\
\hline 10 & 69 & 86 \\
\hline 15 & 88 & 98 \\
\hline 20 & 94 & 100 \\
\hline 25 & 96 & 100 \\
\hline 30 & 100 &
\end{tabular}

(DTP) y el área ocupada por las partículas de cuarzo que han producido grietas en la matriz y que eran observables a 300 aumentos (A-CGM) (partículas diferenciadas por el contraste con respecto a la matriz y las demás partículas). Los resultados se recogen en la Tabla III.

En los dos casos se observan características semejantes tanto para el área ocupada por la porosidad + desconchados como para la distribución de tamaños de partículas de cuarzo que han producido grietas en la matriz. Sin embargo, para el caso de la probeta del enfriamiento más rápido, E6, el área ocupada por las partículas de cuarzo con grietas en la matriz ha sido casi el doble que para la probeta del enfriamiento lento. Estos resultados confirman una vez más la hipótesis que las partículas de cuarzo generan un deterioro microestructural asociado a la diferencia de expansión térmica con la matriz, independientemente de la velocidad de enfriamiento. Sin embargo, a medida que la velocidad de enfriamiento es más elevada (enfriamiento E1 a E6), cuando tiene lugar la transformación alotrópica del cuarzo, las grietas generadas aumentan sus dimensiones más fácilmente, ayudadas por las tensiones térmicas, dando como resultado un deterioro microestructural más severo.

\section{CONCLUSIONES}

En este trabajo se ha comprobado que el gres porcelánico es un producto sensible a un proceso de templado, al desarrollar tensiones residuales macroscópicas durante el enfriamiento rápido típico del ciclo de cocción industrial, y por lo tanto estaría sujeto a las mismas variables de control que un proceso de templado de vidrio.

$\mathrm{Al}$ aumentar el nivel de tensiones residuales, como consecuencia de un enfriamiento más rápido en la zona de comportamiento viscoelástico del material (por encima aproximadamente de $700^{\circ} \mathrm{C}$ ), se incrementa la resistencia mecánica del gres porcelánico, al igual que ocurre en un proceso de templado de vidrio. La existencia de gradientes térmicos entre la superficie y el interior de la pieza durante la transformación alotrópica del cuarzo se traduce en un deterioro microestructural de la pieza que contrarresta el efecto de templado, es decir, la resistencia mecánica no aumenta tanto como cabría esperar.

Las técnicas experimentales para la medida de las tensiones residuales empleadas en esta investigación (relajación de deformaciones por corte incremental y DRX) han mostrado su utilidad y potencialidad para ser empleadas en materiales cerámicos como el gres porcelánico. Son particularmente interesantes a la hora de estudiar con claridad la relación existente entre la microestructura superficial y las propiedades mecánicas.

El desarrollo de perfiles de tensiones residuales en el gres porcelánico no significa necesariamente una ventaja. Si el proceso de enfriamiento del ciclo de cocción no está bien diseñado, se pueden generar grandes fluctuaciones en las características de los materiales obtenidos.

\section{AGRADECIMIENTOS}

Al personal del Instituto de Tecnología Cerámica - ITC, España. A La "Coordenação de Aperfeiçoamento de Pessoal de Nível Superior" - CAPES, Brasil. Al Instituto Maximiliano Gaidzinski - IMG, Brasil. 
Los autores agradecen también la cofinanciación recibida por parte del Ministerio de Industria, Turismo y Comercio, dentro del Programa Horizontal de Apoyo a Centros Tecnológicos (FIT-030000-2005-315/ FIT-030000-2006-119).

\section{BIBLIOGRAFÍA}

1. E. Sánchez. Consideraciones Técnicas Sobre el Producto y el Proceso de Fabricación del Gres Porcelánico. Ceram. Inform, 285, pp. 73-91, 2002.

2. P.M.T Cavalcante, M. Dondi, G. Ercolani, G. Guarini, C. Melandri, M. Raimondo, E.R. Almendra, The Influence of Microstructure on the Performance of White Porcelain Stoneware. Ceram. Int. 30, pp. 953-963, 2004

3. S.L. Correia, D. Hotza, A. M. Segadães. Optimising Mechanical Strength and Bulk Density of Dry Ceramic Bodies Through Mixture Design. Bol. Soc. Esp. Ceram. V., 44 [1], 53-58, 2005.

4. J. Lu. Handbook of Measurement of Residual Stress. Society for Experimental Mechanics, Inc., ed. Fairmont Press, Lilburn - USA, 1996.

5. R.A. McMaster. Fundamentals of Tempered Glass. Ceram.Eng.Sci.Proc. 10 [3-4] pp. 193-206, 1989.

6. J.M.F. Navarro. El Vidrio, Constitución, Fabricación y Propiedades. $3^{\mathrm{a}}$ Edición, CSIC, Madrid-España, 2003.

7. V.M. Sglavo, A. Prezzi, M. Alessandrini. Processing Glasses with Engineered Stress Profiles. J.Non.Cryst.Sol. 344, pp. 73-78, 2004.
8. V.R. Mastelaro, E.D. Zanotto. Anisotropic Residual Stress in Partially Crystallized $\mathrm{Li}_{2} \mathrm{O}-2 \mathrm{SiO}_{2}$ Glass-Ceramics. J. Non-Cryst. Sol. 247, pp. 79-86, 1999

9. U. Senapati, W.M. Carty. Porcelain-Raw Materials, Processing, Phase Evolution, and Mechanical Behavior. J.Am.Ceram.Soc, 81 [1] pp.3-20, 1998.

10. W.M. Carty, B.M. Pinto. Effect of Filler Size on the Strength of Porcelain Bodies, Ceram. Eng.Sci.Proc., 23 [2], pp.95-105, 2002.

11. Y.Takahashi, Y. Ohya. Acoustic Emission from a Porcelain Body During Cooling. J. Am.Ceram.Soc., 82 [2] pp.445-48, 1999.

12. C. Ftikos, C.J. Stournaras, A. Ekonomakou, G. Stathis. Effect of Firing Condition, Filler Grain Size and Quartz Content on Bending Strength and Physical Properties of Sanitaryware Porcelain. J.Eur.Ceram.Soc, 24, pp.23572366, 2004.

13. E.Kato, T. Satoh, O. Ohira, Y. Kobayashi. Effect of Quartz on the Sintering and Bending Strength of the Porcelain Bodies in Quartz-Feldspar-Kaolin System, J. Ceram. Soc. Jap. Int, 102 [1] pp.99-104, 1994.

14. S.R. Bagança, C.P. Bergmann, H. Hübner. Effect of Quartz Particle Size on the Strength of Triaxial Porcelain. J. Eur. Ceram. Soc, 26 [16] pp.2383-2388, 2006.

15. Y. Kobayashi, O. Ohira, Y. Ohashi, E. Kato. Effect of Firing Temperature on Bending Strength of Porcelain for Tableware. J. Am.Ceram.Soc., 75 [7] pp.1801-1806, 1992.

16. R. Bermejo, A.J. Sánchez-Herencia, C. Baudín, L. Llanes, L. Tensiones residuales en cerámicas multicapas de $\mathrm{Al} 2 \mathrm{O} 3-\mathrm{ZrO} 2$ : naturaleza, evaluación y consecuencias sobre la integridad estructural. Bol. Soc. Esp. Ceram. V., 45 [5], 352-354, 2006.

17. C.C. Aydiner, E. Üstündag, M.B. Prime, A. Peker. Modeling and Measurement of Residual Stress in a Bulk Metallic Glass Plate. J.Non.Cryst. Sol. 316, pp. 82-95, 2003.

18. S. R. Stock, B.D. Cullity. Elements of X-Ray Diffraction, Third Edition, Prentice Hall, New Jersey, 2001.

19. K. Kihara. An X-ray Study of the Temperature-Dependence of the Quartz Structure. Quartz. Eur. J. Min., 2, pp. 63-77, 1990.

Recibido: 02.01 .07

Aceptado: 03.04 .07 